\title{
Stacked Ensemble Machine Learning for Range-Separation Parameters
}

Cheng-Wei Ju, ${ }^{\dagger}$ Cheng-Wei Ju, ${ }^{\dagger, \ddagger}$ Ethan J. French, ${ }^{\dagger}$ Ethan J. French, ${ }^{\dagger}$ Nadav Geva, ${ }^{\uparrow}$ Alexander W. Kohn, ${ }^{\S}$ and Zhou Lin*,†

$\dagger$ Department of Chemistry, University of Massachusetts, Amherst, Massachusetts 01003, United States

$\ddagger$ Pritzker School of Molecular Engineering, The University of Chicago, Chicago, Illinois 60637, United States

\Advanced Micro Devices Inc., Boxborough, Massachusetts 01719, United States $\S$ Blizzard Entertainment Inc., Irvine, California 92618, United States

E-mail: zhoulin@umass.edu 


\begin{abstract}
High-throughput virtual materials and drug discovery based on density functional theory has achieved tremendous success in recent decades, but its power on organic semiconducting molecules suffered catastrophically from the self-interaction error until the optimally tuned range-separated hybrid (OT-RSH) exchange-correlation functionals were developed. The accurate but expensive first-principles OT-RSH transitions from a short-range (semi-)local functional to a long-range Hartree-Fock exchange at a distance characterized by the inverse of a molecule-specific, nonempirically-determined range-separation parameter $(\omega)$. In the present study, we proposed a promising stacked ensemble machine learning (SEML) model that provides an accelerated alternative of OT-RSH based on system-dependent structural and electronic configurations. We trained ML- $\omega$ PBE, the first functional in our series, using a database of 1,970 organic semiconducting molecules with sufficient structural diversity, and assessed its accuracy and efficiency using another 1,956 molecules. Compared with the first-principles OT- $\omega$ PBE, our ML- $\omega$ PBE reached a mean absolute error of $0.00504 a_{0}^{-1}$ for the optimal value of $\omega$, reduced the computational cost for the test set by 2.66 orders of magnitude, and achieved comparable predictive powers in various optical properties.
\end{abstract}


Organic semiconducting molecules with large-scale $\pi$-conjugations ${ }^{1-3}$ have been the spotlight of next-generation materials ${ }^{4-8}$ and biological sciences ${ }^{9-13}$ since the 1960 s because of their compelling electronic and optical properties. Due to the ever-growing demand for materials and drug discovery, high-throughput development of organic semiconducting molecules has gradually shifted gear from traditional tedious trial-and-error experiments to first-principles calculations using approaches like density functional theory (DFT). ${ }^{14-19}$ One outstanding example for such a computational design was thermally activated delayed fluorescence (TADF) emitters, for which overall fluorescence quantum yields were predicted based on DFT-evaluated electronic configurations, especially energetic alignment of frontier molecular orbitals. ${ }^{20-22}$

The reliability of DFT can however be impaired by the self-interaction error (SIE) ${ }^{23,24}$ of a (semi-)local exchange-correlation (XC) functional, which leads to an overdelocalized electronic density and an incorrect derivative discontinuity (DD), ${ }^{25-30}$ and eventually a violation of Koopmans ${ }^{\prime 31}$ and Janak's ${ }^{32}$ theorems and an underestimated charge transfer excitation energy. ${ }^{33-36}$ An effective solution is an error-canceling hybrid of the exact Hartree-Fock (HF) exchange energy and some (semi-)local XC functionals, including global hybrids, ${ }^{37-41}$ as well as range-separated hybrids (RSHs) which typically utilize the scheme of ${ }^{34,42-66}$

$$
\frac{1}{\left|\mathbf{r}-\mathbf{r}^{\prime}\right|}=\underbrace{\frac{1-\operatorname{erf}\left(\omega\left|\mathbf{r}-\mathbf{r}^{\prime}\right|\right)}{\left|\mathbf{r}-\mathbf{r}^{\prime}\right|}}_{\mathrm{sr}}+\underbrace{\frac{\operatorname{erf}\left(\omega\left|\mathbf{r}-\mathbf{r}^{\prime}\right|\right)}{\left|\mathbf{r}-\mathbf{r}^{\prime}\right|}}_{\text {lr }}
$$

Here $\left|\mathbf{r}-\mathbf{r}^{\prime}\right|$ represents the interelectron distance and erf represents the Gauss error function. The range-separation parameter $(\omega)$ characterizes the inverse of distance where the shortrange (sr) (semi-)local functional transitions into the long-range (lr) HF exchange. Its ideal value is sensitive to the structural and electronic configuration of a molecule and can be optimally tuned (OT) based on Koopmans' theorem ${ }^{31}$ by minimizing

$$
J^{2}(\omega)=\left[\varepsilon_{\text {HOMO }}(\omega)+I(\omega)\right]^{2}+\left[\varepsilon_{\text {LUMO }}(\omega)+A(\omega)\right]^{2}
$$


where HOMO and LUMO represent the highest occupied and lowest unoccupied molecular orbitals, and $I$ and $A$ describe the ionization potential and the electron affinity, respectively.

OT-RSH functionals possess promoted predictive powers in electronic and optical properties properties, ${ }^{33,67-81}$ but they are expensive because an optimization of $J^{2}(\omega)$ typically requires 20 to 50 converged self-consistent field calculations in the lack of an analytical gradient. Multiple machine learning (ML) strategies have been reported to alleviate the problem. ${ }^{82-95}$ For instance, Chen and coworkers trained long-range-corrected Becke-Lee-Yang-Parr functional using the neural network (LC-BLYP-NN) as the algorithm and atom-atom interactions as molecular descriptors, and minimized the errors of several thermodynamic quantities. ${ }^{82}$ Wang and Zhang constructed a variant of the long-range-corrected Perdew-Burke-Ernzerhof functional based on the localized orbital locator $\left(\mathrm{LC}-\omega \mathrm{PBE} \mathrm{LOL}^{\mathrm{L}}\right)$ that was defined using the kinetic energy, and predicted the singlet-triplet gap $\left(\Delta E_{\mathrm{ST}}\right)$ of metal-free TADF emitters in comparable quality to OT- $\omega$ PBE with a small mean absolute error $(\mathrm{MAE}=0.09 \mathrm{eV}) .{ }^{83}$ Corminboeuf and coworkers designed a ML-based $\omega$ PBE functional that recovered the exact DD using the piecewise relationship between the average energy curvature and the electronic number, and reduced the error of the fundamental gap $\left(E_{\mathrm{g}}\right)$ of large hole-transporting molecular materials from $0.54 \mathrm{eV}$ (by one version of $\mathrm{LC}-\omega \mathrm{PBE}$ with $\omega=0.400 a_{0}^{-1}$ ) to $0.15 \mathrm{eV} .{ }^{84}$ Compared to direct ML predictions of excited state properties, ${ }^{96-100}$ these ML-RSH schemes maintained rigorous solutions of time-(in)dependent Kohn-Sham equations along with valid physical meanings, although they sometime suffered from overfitting and undergeneralization problems due to the limited size and diversity of training sets.

Herein we proposed an "top-down" ML strategy which optimized the molecule-specific $\omega$ in a RSH based on inexpensive molecular descriptors rather than Koopmans' theorem or the energy v.s. electron number curvature. Our representative functional ML- $\omega$ PBE borrowed the formula of $\mathrm{LC}-\omega \mathrm{PBE}^{64}$ but left the value of $\omega$ to be determined by ML algorithms. We will show that ML- $\omega$ PBE reached a comparably strong predictive power to OT- $\omega$ PBE but only consumed a fractional time after complete training. To ensure the high generalizabil- 
ity and accuracy of our model, we were very cautious in deciding the database, molecular descriptors, and algorithms.

For our database we needed molecules that possess rich spectroscopic and photochemical properties but are notorious in theoretical studies due to SIE. We selected 3,926 such species from existing databases, including 1,941 solar cell materials from the Harvard Clean Energy Project (CEP), ${ }^{101,102} 904$ pharmaceutically significant compounds from the DeepChem database, ${ }^{103} 431$ fluorescence species from the ChemFluor database, ${ }^{104} 337$ organic photovoltaic (OPV) molecules from the Harvard Organic Photovoltaic Dataset (HOPV15), ${ }^{105} 84$ organic light-emitting diode (OLED) materials studied by Aspuru-Guzik and coworkers, ${ }^{106}$ and 229 oligomers added by us in the present work. These compounds were randomly distributed into a training set of 1,970 and a test set of 1,956, and their structures were provided as simplified molecular-input line-entry system (SMILES) strings in the Supporting Information (SI). We generated the optimal value of $\omega$ for each molecule using OT- $\omega \mathrm{PBE}$ $\left(\omega_{\mathrm{OT}}\right)$ and provided them as part of the SI. For the training set these $\omega_{\mathrm{OT}}$ 's were included in the training data along with molecular descriptors, while for the test set they were utilized as a reference to calibrate ML- $\omega$ PBE. A wide distribution of these $\omega_{\mathrm{OT}}$ 's was shown to be between $0.120 a_{0}^{-1}$ and $0.330 a_{0}^{-1}$ for the entire database (Fig. 1), with statistics of $\bar{\omega}_{\mathrm{OT}}=(0.206 \pm 0.029) a_{0}^{-1}$. Different databases presented distinct ranges of $\omega_{\mathrm{OT}}$, but only 10 species lied at $\omega_{\mathrm{OT}}>0.300 a_{0}^{-1}$. This result confirmed the diversity of our database but meanwhile invalidated the choice of $\omega=0.300 a_{0}^{-1}$ in the default LC- $\omega \mathrm{PBE}^{64}$ to the majority of organic semiconducting molecules.

To describe important molecular features, we needed a molecular descriptor that captures unique structural and electronic properties. We herein introduced a "composite molecular descriptor" which implements contributions from several singular molecular descriptors like combined molecular fingerprints (CMFs), ${ }^{107-111}$ physical organic descriptors (PODs), ${ }^{108,109}$ and electronic structure properties (ESPs). ${ }^{112-114}$ Detailed information about singular and composite molecular descriptors were provided in the SI. A precursor of our composite molec- 


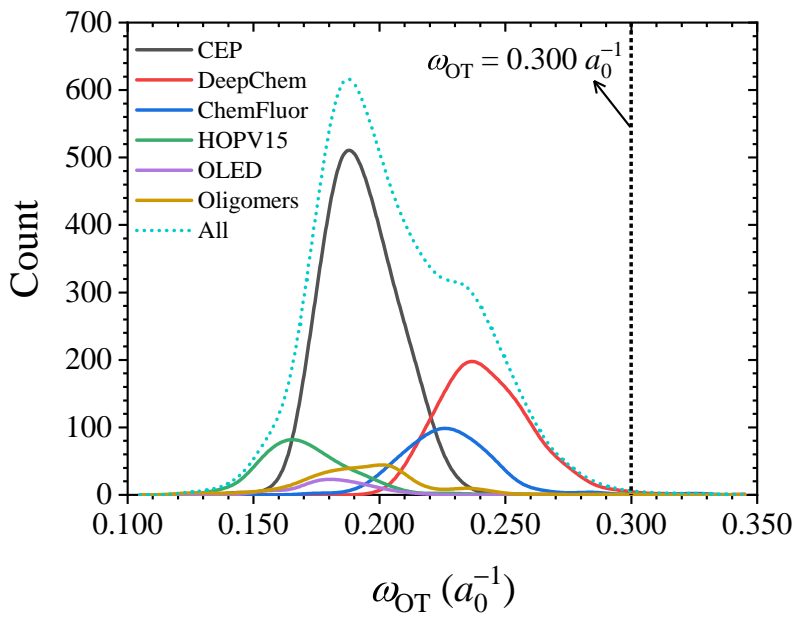

Figure 1: $\omega_{\mathrm{OT}}$ evaluated using Koopmans'-theorem-based OT- $\omega$ PBE for all 3,926 organic semiconducting molecules in the database, labeled by their sources. ${ }^{101-106}$ The default LC$\omega \mathrm{PBE}$ with $\omega=0.300 a_{0}^{-1}$ (vertical dashed line) ${ }^{64}$ is a poor representation for the present dataset.

ular descriptor was designed by Ju and coworkers and proved a success. ${ }^{104}$ In a preliminary model of ML- $\omega \mathrm{PBE}$ which was constructed using the extreme gradient boosting (XGBoost) algorithm, ${ }^{115}$ our composite molecular descriptor $(\mathrm{CMF}+\mathrm{POC}+\mathrm{ESP})$ outperformed all singular or dual molecular descriptors in predicting $\omega_{\mathrm{ML}}$ for the test set (Table S3), exhibiting the lowest mean absolute errors: $\mathrm{MAE}=0.00573 a_{0}^{-1}$ for composite, $\geq 0.00621 a_{0}^{-1}$ for singular and $\geq 0.00577 a_{0}^{-1}$ for dual, with all errors defined as $\Delta=\omega_{\mathrm{ML}}-\omega_{\mathrm{OT}}$. This result endorsed our choice of the composite molecular descriptor.

To enhance the overall predictive power of ML- $\omega$ PBE and future ML-RSH functionals in our series, we desired an algorithm that supersedes simple regression models and artificial neural networks in terms of promoting the predictive power and mitigating the overfitting problem. Earlier methodological studies established the stacked ensemble machine learning (SEML) approach on top of multiple regression methods (base learners) using an overall analytical algorithm (meta learner), and proved them to meet our demands. ${ }^{116-121}$ For our present SEML protocol (Fig. 2), we selected eight base learners, including two versions of least absolute shrinkage and selection operator (LASSO_1 and LASSO_2, differing by molecular descriptors), ${ }^{122,123}$ random forest $(\mathrm{RF}),{ }^{124,125}$ gradient boosted regression trees 
(GBRT),${ }^{126}$ XGBoost, ${ }^{115}$ light gradient boosting machine (LightGBM),${ }^{127}$ kernel ridge regression (KRR), ${ }^{128-131}$ and support vector machine (SVM). ${ }^{132-136}$ The criteria of selecting base learners included (a) their accuracy and (b) their abilities to differentiate between each other. Criterion (a) made sure a base learner provided effective information to the meta learner, and criterion (b) guaranteed that the stacked ensemble learning algorithm improved the predictive power over every single base learner. Therefore, we chose tree-based algorithms, kernel-based algorithms, and linear algorithms. Brief descriptions of algorithms and hyperparameters of these base learners were provided in the SI.

Each selected base learner generated a quantitative relationship between the composite molecular descriptor and the optimal value of $\omega$ though the out-of-fold (OOF) crossvalidation. ${ }^{137}$ After that the least angle regression (LARS) ${ }^{138}$ algorithm was used as the overall meta learner, which analyzed all trained relationships, exploited advantages of all base learners, and optimized the combined results. ${ }^{116-121}$ The algorithm of LARS was also provided in the SI. Given the rate-limiting step of SEML to be the semi-empirical tightbinding (xTB) electronic structure calculation (Fig. 2), the meta learner did not add a significant computational cost to the entire procedure. In a performance analysis of ML$\omega \mathrm{PBE}$ in the accuracy of $\omega_{\mathrm{ML}}$ for the test set (Fig. 3(a)(b) and Table S5), our SEML model was superior to every single base learner by giving the lowest errors in $\omega_{\mathrm{ML}}$ (MAE $\left.=0.00504 a_{0}^{-1}\right)$, the strongest linear correlation between $\omega_{\mathrm{ML}}$ and $\omega_{\mathrm{OT}}\left(R^{2}=0.930^{139}\right)$, as well as the smallest occurrence of large errors: only $13.2 \%$ of molecules showed $|\Delta|>0.010 a_{0}^{-1}$ and $2.6 \%$ presented $|\Delta|>0.020 a_{0}^{-1}$. Compared to OT- $\omega$ PBE, our SEML-based ML- $\omega$ PBE reduced the average computational cost in determining $\omega$ for the test set by 2.66 orders of magnitude, from 41,940 s to $92 \mathrm{~s}$. Our analysis supported the earlier assertion that SEML will surpass all individual base learners regarding applicability and predictability. The source code and database formulated in the present study were provided in the GitHub repository of the Lin Group. ${ }^{140}$

Following the discussions of the ML strategy and the accuracy of $\omega_{\mathrm{ML}}$, we will validate 


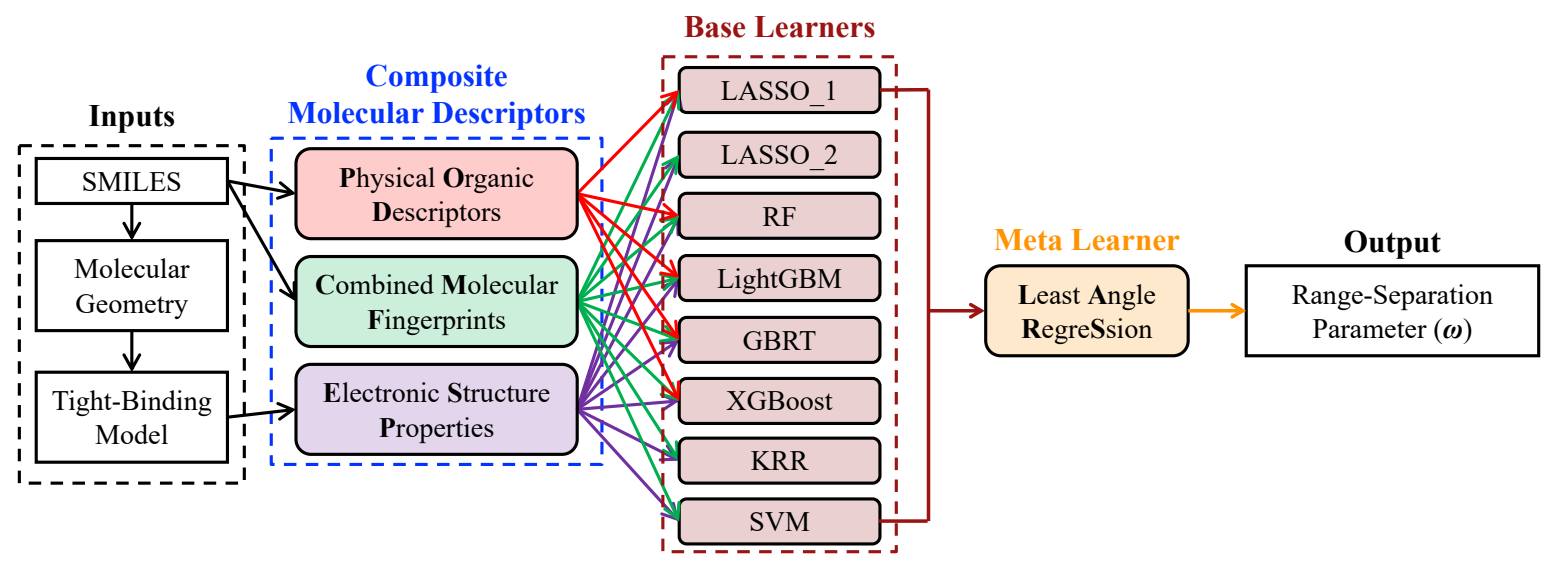

Figure 2: Schematic illustration for our SEML protocol. ${ }^{116-121}$ The model reads structural and electronic properties, translates them into composite molecular descriptors, ${ }^{108,109,112-114}$ and optimizes $\omega$ based on eight base learners ${ }^{115,122-127,131,135}$ and the meta learner of LARS. ${ }^{138}$

the versatility of ML- $\omega \mathrm{PBE}$ in high-throughput materials and drug discovery by showing that its generalizability and reliability is quantitatively comparable to OT- $\omega \mathrm{PBE}^{141}$ and is more substantial than other popular functionals, including four non-tuned RSH functionals, LC- $\omega$ PBE with $\omega=0.300 a_{0}^{-1},{ }^{64}$ CAM-B3LYP,${ }^{47}$ M06-2X, ${ }^{142}$ and $\omega$ B97X-D3 ${ }^{54}$ and four non-RSH functionals, HF, ${ }^{143} \mathrm{PBE},{ }^{144} \mathrm{PBE} 0,{ }^{40,41}$ and B3LYP. ${ }^{37-39}$ We will evaluate their performances on multiple experimentally observables, including $E_{g}$, the vertical absorption, fluorescence, and phosphorescence energies $\left(E_{\mathrm{abs}}, E_{\mathrm{fl}}\right.$, and $\left.E_{\mathrm{ph}}\right), \Delta E_{\mathrm{ST}}$, and the fluorescence transition dipole moment and lifetime $\left(\mu_{\mathrm{fl}}\right.$ and $\left.\tau_{\mathrm{fl}}{ }^{145}\right)$. We selected molecules with corresponding experimental measurements as the test of of optical properties. and categorized them into subsets based on their structural features, excited-state properties, and real-life applications. These subsets include OPV $,{ }^{141,146} \mathrm{TADF},{ }^{147}$ polycyclic aromatic hydrocarbons (PAH), ${ }^{141}$ aggregation-induced emission molecules (AIE), ${ }^{148}$ fluorophores (FL) ${ }^{149}$ and bio-organic molecules (BIO). ${ }^{141}$ The majority of molecules in the present test set of optical properties, such as all AIE compounds and many OPV and TADF structures, were not included in the above-mentioned test set of $\omega_{\mathrm{ML}}$, so that they served as great benchmarks for the generalizability of our model. The statistics of errors, defined based on the signed differences between calculated and experimental values, are summarized in Figs. 4 and S2, 
(a)

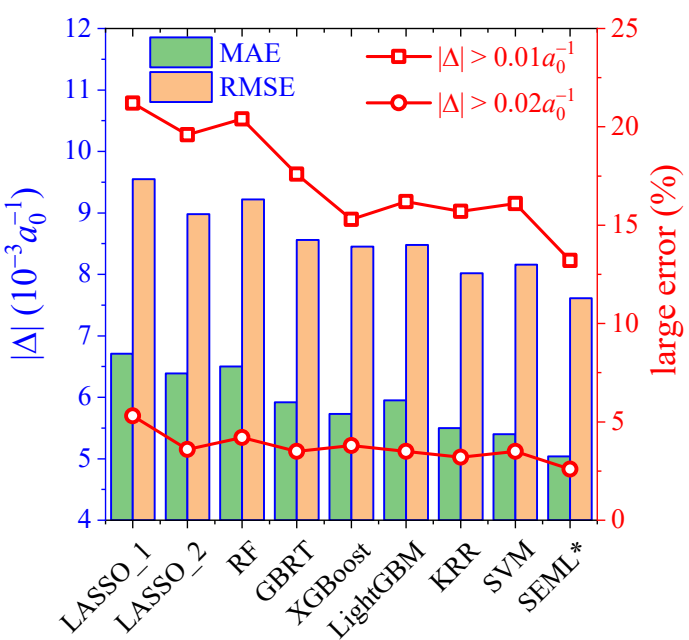

Algorithm

(b)

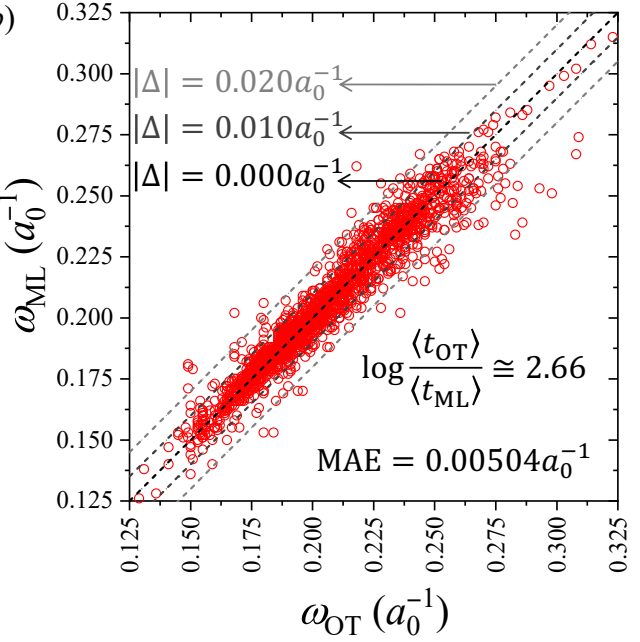

Figure 3: (a) Comparison in the error of $\omega_{\mathrm{ML}}(\Delta)$ optimized using eight base learners and the SEML algorithm. (b) Comparison between $\omega_{\mathrm{OT}}$ (x-axis) and $\omega_{\mathrm{ML}}$ ( $y$-axis) with labels of $|\Delta|=0.000$ (black line), $0.010 a_{0}^{-1}$ (dark gray lines) and $0.020 a_{0}^{-1}$ (light gray lines). Our SEML-based ML- $\omega$ PBE presents the lowest MAE of $0.00504 a_{0}^{-1}$ and the lowest RMSE of $0.00761 a_{0}^{-1}$, as well as the smallest percentages for large errors $\left(13.2 \%\right.$ for $|\Delta|>0.010 a_{0}^{-1}$ and $2.6 \%$ for $\left.|\Delta|>0.020 a_{0}^{-1}\right)$. The average computational cost of ML- $\omega$ PBE is 2.66 orders of magnitude smaller than OT- $\omega \mathrm{PBE}$. 
as well as Tables S6 through S9.

$E_{\mathrm{g}}$ is the most straightforward observable to assess because it is the difference between two important eigenvalues that are relevant to Koopmans' theorem, $\varepsilon_{\text {HOMO }}$ and $\varepsilon_{\text {LUMO }}$ (Eq. (2)), and is susceptible to the quality of the asymptotic density decay. As we expected (Fig. 4 and Table S6), non-RSH functionals that had been successful on small organic molecules were no longer accurate for medium and large organic semiconducting molecules (MAE $\geq 2.401$ $\mathrm{eV}$ ), while the performance of a RSH functional was more acceptable (MAE $\leq 1.209 \mathrm{eV}$ ) especially ML- $\omega \mathrm{PBE}(\mathrm{MAE}=0.681 \mathrm{eV})$ and OT- $\omega \mathrm{PBE}(\mathrm{MAE}=0.733 \mathrm{eV})$. The advantage was magnified for molecules with large spatial extents of $\pi$-conjugations like OPV (MAE $=0.441 \mathrm{eV}$, Fig. S3(a)) and PAH (MAE $=0.271 \mathrm{eV}$, Fig. S3(b)) whose energetics are keen to the choice of $\omega$, but was less obvious for smaller $\pi$-bonds like BIO (MAE $=1.870 \mathrm{eV}$, Fig. S3(c)) without fitting to experimental values. The surprisingly better behavior of ML$\omega \mathrm{PBE}$ than OT- $\omega \mathrm{PBE}$ was probably due to the bias from the incomplete test subset or the cancellation between systematic and random errors, but it did not damage our conclusion about the accuracy of ML- $\omega$ PBE.

In spite of weaker relevance to Koopmans' theorem, optical band gaps $E_{\mathrm{abs}}, E_{\mathrm{fl}}$, and $E_{\mathrm{ph}}$ are more interesting benchmark quantities because they have more abundant and reliable experimental data from UV-vis spectra and their accurate predictions rely on electron-hole interactions in addition to asymptotic properties. ${ }^{141}$ They were all evaluated using linearresponse time-dependent DFT (LR-TDDFT), ${ }^{150-152}$ with $E_{\text {abs }}$ at the ground state geometries where the training occurred and $E_{\mathrm{fl}}$ and $E_{\mathrm{ph}}$ at corresponding excited state geometries. For these three optical band gaps, although hybrid non-RSH functionals like PBE0 ${ }^{40,41}$ and B3LYP ${ }^{38,39}$ can outperform non-tuned RSHs due to error cancellation between densityoverlocalized HF and density-overdelocalized (semi-)local functionals, they were generally less accurate than ML- $\omega \mathrm{PBE}$ and OT- $\omega \mathrm{PBE}$. ML- $\omega \mathrm{PBE}$ illustrated the lowest MAE of $0.194 \mathrm{eV}$ for $E_{\mathrm{ff}}$ and the second-lowest MAEs of $0.182 \mathrm{eV}$ and $0.206 \mathrm{eV}$ for $E_{\mathrm{abs}}$ and $E_{\mathrm{ph}}$, respectively. These errors were only marginally different from OT- $\omega$ PBE which provided 


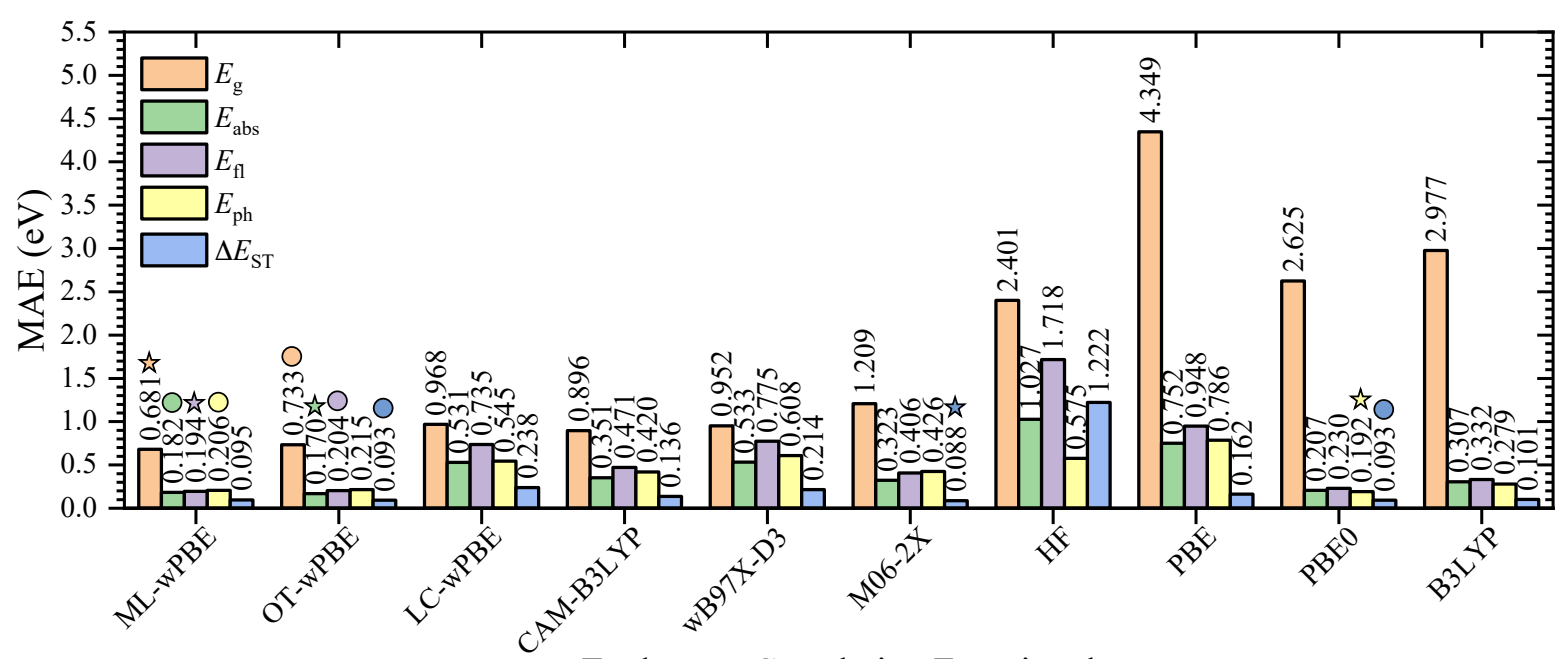

Exchange-Correlation Functional

Figure 4: MAEs of $E_{\mathrm{g}}$ (orange), $E_{\mathrm{abs}}$ (green), $E_{\mathrm{fl}}$ (violet), $E_{\mathrm{ph}}$ (yellow), and $\Delta E_{\mathrm{ST}}$ (blue) (all in $\mathrm{eV}$ ) were compared across various $\mathrm{XC}$ functionals for selected molecules in the test set. The lowest and second-lowest MAEs were labeled with stars and circles respectively. For these organic semiconducting molecules, ML- $\omega$ PBE exhibits a quantitatively comparable accuracy to OT- $\omega$ PBE, especially for molecules with large $\pi$-conjugations and charge-transfer excited states.

MAEs of $0.170 \mathrm{eV}, 0.204 \mathrm{eV}$, and $0.215 \mathrm{eV}$ for $E_{\mathrm{abs}}, E_{\mathrm{fl}}$, and $E_{\mathrm{ph}}$, respectively (Fig. 4 and Tables S7 and S8). Interestingly, just like OT- $\omega$ PBE, ML- $\omega$ PBE minimized the systematic errors and made the overall errors more evenly distributed around zero, showing the second lowest MSEs of $+0.106 \mathrm{eV},+0.109 \mathrm{eV}$, and $+0.115 \mathrm{eV}$ for $E_{\mathrm{abs}}, E_{\mathrm{fl}}$, and $E_{\mathrm{ph}}$. This discovery confirmed that ML- $\omega$ PBE trained over ground state configurations are robustly generalizable to low-lying excited state structures.

Among all test species, subsets of TADF exhibited MAEs of $0.139 \mathrm{eV}, 0.194 \mathrm{eV}$, and 0.206 $\mathrm{eV}$ from ML- $\omega \mathrm{PBE}$ for $E_{\mathrm{abs}}, E_{\mathrm{fl}}$, and $E_{\mathrm{ph}}$, respectively, (Fig. S3(d)) and AIE showed MAE $=$ $0.239 \mathrm{eV}$ from ML- $\omega \mathrm{PBE}$ for $E_{\text {abs, }}$ (Fig. S3(e)). They illustrated apparent preference towards ML- $\omega$ PBE and OT- $\omega$ PBE because their HOMO and LUMO are located at two different parts of the molecule or complex, which stretches the electronic density to the asymptotic limit and is stable over slightly varied local electron-hole interaction. The result of the AIE subset was of particular interest because it indicated a great potential to extend our model from single molecules to molecular complexes or aggregates without extra treatment, even when 
no molecular complexes and aggregates were included in the training set. On the contrary, HOMO and LUMO for the OPV subset (with MAE $=0.147 \mathrm{eV}$ from ML- $\omega \mathrm{PBE}$ for $E_{\text {abs }}$, Fig. S3(a)) are greatly overlapped in space and emphasizes the subtlety in the electronhole interaction, allowing the error-canceling PBE0 to win. In addition to the argument about orbital configurations, the large discrepancy between ML- $\omega$ PBE and OT- $\omega$ PBE in OPV indicates a second possible but resolvable origin of the error: a poor prediction of $\omega$ in ML- $\omega$ PBE due to the lack of electron acceptors in the training set.

In addition to optical band gaps, $\Delta E_{\mathrm{ST}}$ is an essential property for the TADF subset because it is explicitly correlated to the quantum yield of TADF ${ }^{147,153}$ and is highly responsive to the extent of charge separation. $\Delta E_{\mathrm{ST}}$ exhibited a different but understandable trend from $E_{\mathrm{fl}}$ or $E_{\mathrm{ph}}$ (Fig. 4 and Table S8): many functionals that had significantly overestimated $E_{\mathrm{fl}}$ and $E_{\mathrm{ph}}$ (like M06-2X) or underestimated them (like PBE) predicted equally accurate $\Delta E_{\mathrm{ST}}$ 's to ML- $\omega \mathrm{PBE}$ and $\mathrm{OT}-\omega \mathrm{PBE}$ due to the cancellation in systematic error between singlet and triplet states. Such accidentally good results with other functionals, again, do not compromise the advantage of ML- $\omega$ PBE.

Finally, we benchmarked the performance of ML- $\omega$ PBE in $\mu_{\mathrm{fl}}$ and $\tau_{\mathrm{fl}}$, which are key quantities to determine difficult-to-evaluate composite photodynamic properties like the fluorescence quantum yield ${ }^{18,19}$ and the luminescence dissymmetry factor. ${ }^{154,155}$ We selected only five RSH functionals in our comparison due to their successful descriptions of electronic density distributions (Fig. S4(a)(b) and Table S9), and evaluated both $\mu_{\mathrm{fl}}$ and $\tau_{\mathrm{fl}}$ using LRTDDFT. Because of a successful prediction of excited state electronic density, ML- $\omega$ PBE provided almost equally accurate prediction to OT- $\omega \mathrm{PBE}$ for both $\mu_{\mathrm{fl}}$ (MAE $=0.830$ debye versus 0.827 debye) and $\tau_{\mathrm{fl}}(\mathrm{MAE}=1.89 \mathrm{~ns}$ versus $1.90 \mathrm{~ns})$, and outperformed other $\mathrm{RSH}$ functionals. This result allowed us to safely assert that ML- $\omega$ PBE best described photodynamic properties in the LR framework. However, because the LR approximation is not able to capture large electronic rearrangement during charge-transfer transitions, it might end up with a poor emissive geometry and might introduce a systematic error that consistently 
overestimates $\mu_{\mathrm{fl}}$ while underestimating $\tau_{\mathrm{fl}}$. This problem explained the sizable MSEs across all functionals and cannot be resolved without methodological treatment. ${ }^{156-159}$

To conclude the present study, our ML- $\omega$ PBE functional was established using a topdown SEML strategy based on eight base learners and the LARS meta learner, and it can accurately and efficiently reproduce the molecule-specific formula of Koopmans' theorembased OT- $\omega$ PBE using computationally inexpensive structural and electronic properties, as well as theoretically challenging optical properties using low-cost DFT and LR-TDDFT. To make sure of sufficient reliability and generalizability for our SEML method, we selected 3,926 structurally diverse and experimentally well-studied organic semiconducting molecules into our training and test sets, and extracted their composite molecular descriptors based on structural analyses and the semi-empirical xTB calculations. After a two-step training through base and meta learners, we constructed an explicit map between $\omega_{\mathrm{ML}}$ and the composite molecular descriptor, and obtained an outstanding agreement between $\omega_{\mathrm{ML}}$ and $\omega_{\mathrm{OT}}{ }^{141}$ for the test set, with an MAE of $0.00504 a_{0}^{-1}$ and a shorter timescale than OT- $\omega$ PBE by 2.66 orders of magnitude.

To further evaluate the quality of ML- $\omega \mathrm{PBE}$, we compared its performance on a few experimental observable optical properties with OT- $\omega \mathrm{PBE}$, four non-tuned RSH functionals, and four non-RSH functionals. Taking into account all possible error cancellations, the fully trained ML- $\omega$ PBE reached a wonderful performance that was quantitatively analogous to OT- $\omega$ PBE in all optical properties, and surpassed every single non-RSH and non-tuned RSH functional. The fact that ML- $\omega$ PBE was trained based solely on ground state properties and monomeric molecules did not weaken its predictive power on excited state properties and molecular aggregates or complexes. Our result validated the practical usefulness of ML$\omega \mathrm{PBE}$ in reproducing and predicting optical properties for real-life materials and drugs. In conclusion, the present study did not only propose an accurate and efficient way to determine selected parameters in the XC functional, but also expanded the horizon of ML applications in the framework of quantum chemistry. We expected adequately trained ML- 
$\omega \mathrm{PBE}$ and future ML-RSH functionals to replace first-principles OT-RSHs in large-scale virtual materials and drug discovery to overcome the bottleneck in the computational cost.

Supporting Information The Supporting Information is available free of charge on the ACS Publications website at DOI: XXX/XXXXXX. SMILES strings and $\omega$ values for all 3,926 molecules in the training and test sets, brief proof of Koopmans' theorem and asymptotic decay of electronic density, descriptions of details for general OT- $\omega$ PBE and ML$\omega \mathrm{PBE}$ functionals and quantum chemical calculations, composite molecular descriptors, and base and metal learners in the SEML model, and summaries of error statistics of ML- $\omega$ PBE and other $\mathrm{XC}$ functionals in optical properties (PDF). Link to the source code and database on the GitHub repository of the Lin Group: https://github.com/zhoulinumass/ML-wPBE.

Acknowledgments Z. L. would like to thank the University of Massachusetts Amherst for offering start-up funds and the Massachusetts Green High Performance Computing Center (MGHPCC) for providing computational resources. Z. L., C.-W. J. and E. J. F. would also like to thank Ms. Lixue Cheng, Prof. Hui Guan, Dr. Kun Yao, Dr. Yuezhi Mao, and Mr. Junjie Yang for scientific insights and discussions, as well as Mr. Junyi Gong for providing the benchmarked solvent-based data of the AIE subset.

\section{References}

(1) Kallmann, H.; Pope, M. Surface-Controlled Bulk Conductivity in Organic Crystals. Nature 1960, 185, 753-753.

(2) Shirakawa, H.; Louis, E. J.; MacDiarmid, A. G.; Chiang, C. K.; Heeger, A. J. Synthesis of Electrically Conducting Organic Polymers: Halogen Derivatives of Polyacetylene, $(\mathrm{CH})_{x}$. J. Chem. Soc., Chem. Commun. 1977, 578-580.

(3) Diaz, A. F.; Kanazawa, K. K.; Gardini, G. P. Electrochemical Polymerization of Pyrrole. J. Chem. Soc., Chem. Commun. 1979, 635-636. 
(4) Yang, Z.; Mao, Z.; Xie, Z.; Zhang, Y.; Liu, S.; Zhao, J.; Xu, J.; Chi, Z.; Aldred, M. P. Recent Advances in Organic Thermally Activated Delayed Fluorescence Materials. Chem. Soc. Rev. 2017, 46, 915-1016.

(5) Un, H.-I.; Wang, J.-Y.; Pei, J. Recent Efforts in Understanding and Improving the Nonideal Behaviors of Organic Field-Effect Transistors. Adv. Sci. 2019, 6, 1900375.

(6) Han, L.; Chen, S.; Deng, L.; Song, Y.; Huang, Y.; Li, S.; Li, M.; Tang, W.; Su, Y.; Guo, X. Through-Plastic-Via Three-Dimensional Integration for Integrated Organic Field-Effect Transistor Bio-Chemical Sensor Chip. IEEE Electron Device Lett. 2021, 42, 569-572.

(7) Inganäs, O. Organic Photovoltaics over Three Decades. Adv. Mater. 2018, 30, 1800388.

(8) Kuehne, A. J. C.; Gather, M. C. Organic Lasers: Recent Developments on Materials, Device Geometries, and Fabrication Techniques. Chem. Rev. 2016, 116, 12823-12864.

(9) Westphal, V.; Rizzoli, S. O.; Lauterbach, M. A.; Kamin, D.; Jahn, R.; Hell, S. W. Video-Rate Far-Field Optical Nanoscopy Dissects Synaptic Vesicle Movement. Science 2008, 320, 246-249.

(10) Wang, C.; Taki, M.; Kajiwara, K.; Wang, J.; Yamaguchi, S. Phosphole-Oxide-Based Fluorescent Probe for Super-resolution Stimulated Emission Depletion Live Imaging of the Lysosome Membrane. ACS Mater. Lett. 2020, 2, 705-711.

(11) Hu, F.; Shi, L.; Min, W. Biological Imaging of Chemical Bonds by Stimulated Raman Scattering Microscopy. Nat. Methods 2019, 16, 830-842.

(12) Prince, R. C.; Frontiera, R. R.; Potma, E. O. Stimulated Raman Scattering: From Bulk to Nano. Chem. Rev. 2017, 117, 5070-5094. 
(13) Miao, Y.; Shi, L.; Hu, F.; Min, W. Probe Design for Super-Multiplexed Vibrational Imaging. Phys. Biol. 2019, 16, 041003.

(14) Jacquemin, D.; Perpète, E. A.; Scuseria, G. E.; Ciofini, I.; Adamo, C. TD-DFT Performance for the Visible Absorption Spectra of Organic Dyes: Conventional Versus Long-Range Hybrids. J. Chem. Theory Comput. 2008, 4, 123-135.

(15) Brédas, J.-L.; Norton, J. E.; Cornil, J.; Coropceanu, V. Molecular Understanding of Organic Solar Cells: the Challenges. Acc. Chem. Res. 2009, 42, 1691-1699.

(16) Jacquemin, D.; Wathelet, V.; Perpete, E. A.; Adamo, C. Extensive TD-DFT Benchmark: Singlet-Excited States of Organic Molecules. J. Chem. Theory Comput. 2009, 5, 2420-2435.

(17) Jacquemin, D.; Perpète, E. A.; Ciofini, I.; Adamo, C. Assessment of Functionals for TD-DFT Calculations of Singlet-Triplet Transitions. J. Chem. Theory Comput. 2010, 6, 1532-1537.

(18) Kohn, A. W.; Lin, Z.; Van Voorhis, T. Toward Prediction of Nonradiative Decay Pathways in Organic Compounds I: The Case of Naphthalene Quantum Yields. J. Phys. Chem. C 2019, 123, 15394-15402.

(19) Lin, Z.; Kohn, A. W.; Van Voorhis, T. Toward Prediction of Nonradiative Decay Pathways in Organic Compounds II: Two Internal Conversion Channels in BODIPYs. J. Phys. Chem. C 2020, 124, 3925-3938.

(20) Uoyama, H.; Goushi, K.; Shizu, K.; Nomura, H.; Adachi, C. Highly Efficient Organic Light-Emitting Diodes from Delayed Fluorescence. Nature 2012, 492, 234-238.

(21) Endo, A.; Sato, K.; Yoshimura, K.; Kai, T.; Kawada, A.; Miyazaki, H.; Adachi, C. Efficient Up-Conversion of Triplet Excitons into a Singlet State and Its Application for Organic Light Emitting Diodes. Appl. Phys. Lett. 2011, 98, 083302. 
(22) Tao, Y.; Yuan, K.; Chen, T.; Xu, P.; Li, H.; Chen, R.; Zheng, C.; Zhang, L.; Huang, W. Thermally Activated Delayed Fluorescence Materials Towards the Breakthrough of Organoelectronics. Adv. Mater. 2014, 26, 7931-7958.

(23) Perdew, J. P.; Zunger, A. Self-Interation Correction to Density-Functional Approximations for Many-Electron Systems. Phys. Rev. B 1981, 23, 5048.

(24) Bao, J. L.; Gagliardi, L.; Truhlar, D. G. Self-Interation Error in Density Functional Theory: An Appraisal. J. Phys. Chem. Lett. 2018, 9, 2353-2358.

(25) Perdew, J. P.; Parr, R. G.; Levy, M.; Balduz, J. L. Density-Functional Theory for Fractional Particle Number: Derivative Discontinuities of the Energy. Phys. Rev. Lett. 1982, 49, 1691-1694.

(26) Perdew, J. P.; Levy, M. Physical Content of the Exact Kohn-Sham Orbital Energies: Band Gaps and Derivative Discontinuities. Phys. Rev. Lett. 1983, 51, 1884.

(27) Cohen, A. J.; Mori-Sánchez, P.; Yang, W. Fractional Charge Perspective on the Band Gap in Density-Functional Theory. Phys. Rev. B 2008, 77, 115123.

(28) Yang, W.; Cohen, A. J.; Mori-Sánchez, P. Derivative Discontinuity, Bandgap and Lowest Unoccupied Molecular Orbital in Density Functional Theory. J. Chem. Phys. 2012, 136, 204111.

(29) Chai, J.-D.; Chen, P.-T. Restoration of the Derivative Discontinuity in Kohn-Sham Density Functional Theory: An Efficient Scheme for Energy Gap Correction. Phys. Rev. Lett. 2013, 110, 033002.

(30) Mori-Sánchez, P.; Cohen, A. J. The Derivative Discontinuity of the ExchangeCorrelation Functional. Phys. Chem. Chem. Phys. 2014, 16, 14378-14387.

(31) Koopmans, T. Über die Zuordnung von Wellenfunktionen und Eigenwerten zu den Einzelnen Elektronen Eines Atoms. Physica 1934, 1, 104-113. 
(32) Janak, J. F. Proof that $\frac{\partial E}{\partial n_{i}}=\epsilon$ in Density-Functional Theory. Phys. Rev. B 1978, 18, 7165-7168.

(33) Kronik, L.; Stein, T.; Refaely-Abramson, S.; Baer, R. Excitation Gaps of FiniteSized Systems from Optimally Tuned Range-Separated Hybrid Functionals. J. Chem. Theory Comput. 2012, 8, 1515-1531.

(34) Dreuw, A.; Weisman, J. L.; Head-Gordon, M. Long-Range Charge-Transfer Excited States in Time-Dependent Density Functional Theory Require Non-Local Exchange. J. Chem. Phys. 2003, 119, 2943-2946.

(35) Dreuw, A.; Head-Gordon, M. Failure of Time-Dependent Density Functional Theory for Long-Range Charge-Transfer Excited States: the ZincbacteriochlorinBacteriochlorin and Bacteriochlorophyll-Spheroidene Complexes. J. Am. Chem. Soc. 2004, 126, 4007-4016.

(36) Dreuw, A.; Head-Gordon, M. Single-Eeference Ab Initio Methods for the Calculation of Excited States of Large Molecules. Chem. Rev. 2005, 105, 4009-4037.

(37) Becke, A. D. Density-Functional Exchange-Energy Approximation with Correct Asymptotic Behavior. Phys. Rev. A 1988, 38, 3098-3100.

(38) Lee, C.; Yang, W.; Parr, R. G. Development of the Colle-Salvetti Correlation-Energy Formula into a Functional of the Electron Density. Phys. Rev. B 1988, 37, 785-789.

(39) Becke, A. D. A New Mixing of Hartree-Fock and Local Density-Functional Theories. J. Chem. Phys. 1993, 98, 1372-1377.

(40) Perdew, J. P.; Ernzerhof, M.; Burke, K. Rationale for Mixing Exact Exchange with Density Functional Approximations. J. Chem. Phys. 1996, 105, 9982-9985.

(41) Adamo, C.; Barone, V. Toward Reliable Density Functional Methods without Adjustable Parameters: The PBE0 Model. J. Chem. Phys. 1999, 110, 6158-6170. 
(42) Iikura, H.; Tsuneda, T.; Yanai, T.; Hirao, K. A Long-Range Correction Scheme for Generalized-Gradient-Approximation Exchange Functionals. J. Chem. Phys. 2001, $115,3540-3544$.

(43) Heyd, J.; Peralta, J. E.; Scuseria, G. E.; Martin, R. L. Energy Band Gaps and Lattice Parameters Evaluated with the Heyd-Scuseria-Ernzerhof Screened Hybrid Functional. J. Chem. Phys. 2005, 123, 174101.

(44) Song, J.-W.; Hirosawa, T.; Tsuneda, T.; Hirao, K. Long-Range Corrected Density Functional Calculations of Chemical Reactions: Redetermination of Parameter. J. Chem. Phys. 2007, 126, 154105.

(45) Heyd, J.; Scuseria, G. E.; Ernzerhof, M. Hybrid Functionals Based on a Screened Coulomb Potential. J. Chem. Phys. 2003, 118, 8207-8215.

(46) Henderson, T. M.; Janesko, B. G.; Scuseria, G. E. Generalized Gradient Approximation Model Exchange Holes for Range-Separated Hybrids. J. Chem. Phys. 2008, 128, 194105.

(47) Yanai, T.; Tew, D. P.; Handy, N. C. A New Hybrid Exchange-Correlation Functional Using the Coulomb-Attenuating Method (CAM-B3LYP). Chem. Phys. Lett. 2004, 393, 51-57.

(48) Gerber, I. C.; Ángyán, J. G. Hybrid Functional with Separated Range. Chem. Phys. Lett. 2005, 415, 100-105.

(49) Toulouse, J.; Colonna, F.; Savin, A. Short-Range Exchange and Correlation Energy Density Functionals: Beyond the Local-Density Approximation. J. Chem. Phys. 2005, 122, 014110.

(50) Toulouse, J.; Colonna, F.; Savin, A. Long-Range-Short-Range Separation of the 
Electron-Electron Interaction in Density-Functional Theory. Phys. Rev. A 2004, 70, 062505 .

(51) Chai, J.-D.; Head-Gordon, M. Long-Range Corrected Double-Hybrid Density Functionals. J. Chem. Phys. 2009, 131, 174105.

(52) Chai, J.-D.; Head-Gordon, M. Systematic Optimization of Long-Range Corrected Hybrid Density Functionals. J. Chem. Phys. 2008, 128, 084106.

(53) Akinaga, Y.; Ten-no, S. Range-Separation by the Yukawa Potential in Long-Range Corrected Density Functional Theory with Gaussian-Type Basis Functions. Chem. Phys. Lett. 2008, 462, 348-351.

(54) Lin, Y.-S.; Li, G.-D.; Mao, S.-P.; Chai, J.-D. Long-Range Corrected Hybrid Density Functionals with Improved Dispersion Corrections. J. Chem. Theory Comput. 2013, 9, 263-272.

(55) Seth, M.; Ziegler, T. Range-Separated Exchange Functionals with Slater-Type Functions. J. Chem. Theory Comput. 2012, 8, 901-907.

(56) Mardirossian, N.; Head-Gordon, M. wB97X-V: A 10-Parameter, Range-Separated Hybrid, Generalized Gradient Approximation Density Functional with Nonlocal Correlation, Designed by a Survival-of-the-Fittest Strategy. Phys. Chem. Chem. Phys. 2014, 16, 9904-9924.

(57) Mardirossian, N.; Head-Gordon, M. wB97M-V: A Combinatorially Optimized, RangeSeparated Hybrid, meta-GGA Density Functional with VV10 Nonlocal Correlation. J. Chem. Phys. 2016, 144, 214110.

(58) Peverati, R.; Truhlar, D. G. Improving the Accuracy of Hybrid meta-GGA Density Functionals by Range Separation. J. Phys. Chem. Lett. 2011, 2, 2810-2817. 
(59) Peverati, R.; Truhlar, D. G. An Improved and Broadly Accurate Local Approximation to the Exchange-Correlation Density Functional: The MN12-L Functional for Electronic Structure Calculations in Chemistry and Physics. Phys. Chem. Chem. Phys. 2012, 14, 13171-13174.

(60) Peverati, R.; Truhlar, D. G. M11-L: A Local Density Functional That Provides Improved Accuracy for Electronic Structure Calculations in Chemistry and Physics. J. Phys. Chem. Lett. 2012, 3, 117-124.

(61) Peverati, R.; Truhlar, D. G. Screened-Exchange Density Functionals with Broad Accuracy for Chemistry and Solid-State Physics. Phys. Chem. Chem. Phys. 2012, 14, $16187-16191$.

(62) Peverati, R.; Truhlar, D. G. Exchange-Correlation Functional with Good Accuracy for Both Structural and Energetic Properties while Depending Only on the Density and Its Gradient. J. Chem. Theory Comput. 2012, 8, 2310-2319.

(63) Peverati, R.; Zhao, Y.; Truhlar, D. G. Generalized Gradient Approximation That Recovers the Second-Order Density-Gradient Expansion with Optimized Across-theBoard Performance. J. Phys. Chem. Lett. 2011, 2, 1991-1997.

(64) Rohrdanz, M. A.; Herbert, J. M. Simultaneous Benchmarking of Ground- and ExcitedState Properties with Long-Range-Corrected Density Functional Theory. J. Chem. Phys. 2008, 129, 034107.

(65) Janesko, B. G.; Henderson, T. M.; Scuseria, G. E. Long-Range-Corrected Hybrids Including Random Phase Approximation Correlation. J. Chem. Phys. 2009, 130, 081105 .

(66) Rohrdanz, M. A.; Martins, K. M.; Herbert, J. M. A Long-Range-Corrected Density Functional that Performs Well for Both Ground-State Properties and Time-Dependent 
Density Functional Theory Excitation Energies, Including Charge-Transfer Excited States. J. Chem. Phys. 2009, 130, 054112.

(67) Livshits, E.; Baer, R. A Well-Tempered Density Functional Theory of Electrons in Molecules. Phys. Chem. Chem. Phys. 2007, 9, 2932-2941.

(68) Autschbach, J. Charge-Transfer Excitations and Time-Dependent Density Functional Theory: Problems and Some Proposed Solutions. ChemPhysChem 2009, 10, 17571760.

(69) Stein, T.; Kronik, L.; Baer, R. Reliable Prediction of Charge Transfer Excitations in Molecular Complexes Using Time-Dependent Density Functional Theory. J. Am. Chem. Soc. 2009, 131, 2818-2820.

(70) Stein, T.; Eisenberg, H.; Kronik, L.; Baer, R. Fundamental Gaps in Finite Systems from Eigenvalues of a Generalized Kohn-Sham Method. Phys. Rev. Lett. 2010, 105, 266802.

(71) Baer, R.; Livshits, E.; Salzner, U. Tuned Range-Separated Hybrids in Density Functional Theory. Annu. Rev. Phys. Chem. 2010, 61, 85-109.

(72) Kuritz, N.; Stein, T.; Baer, R.; Kronik, L. Charge-Transfer-Like $\pi \rightarrow \pi^{*}$ Excitations in Time-Dependent Density Functional Theory: A Conundrum and Its Solution. J. Chem. Theory Comput. 2011, 7, 2408-2415.

(73) Refaely-Abramson, S.; Sharifzadeh, S.; Govind, N.; Autschbach, J.; Neaton, J. B.; Baer, R.; Kronik, L. Quasiparticle Spectra from a Nonempirical Optimally Tuned Range-Separated Hybrid Density Functional. Phys. Rev. Lett. 2012, 109, 226405.

(74) Körzdörfer, T.; Parrish, R. M.; Marom, N.; Sears, J. S.; Sherrill, C. D.; Brédas, J.-L. Assessment of the Performance of Tuned Range-Separated Hybrid Density Functionals in Predicting Accurate Quasiparticle Spectra. Phys. Rev. B 2012, 86, 205110. 
(75) Karolewski, A.; Kronik, L.; Kümmel, S. Using Optimally Tuned Range Separated Hybrid Functionals in Ground-State Calculations: Consequences and Caveats. J. Chem. Phys. 2013, 138, 204115.

(76) Autschbach, J.; Srebro, M. Delocalization Error and "Functional Tuning" in KohnSham Calculations of Molecular Properties. Acc. Chem. Res. 2014, 47, 2592-2602.

(77) Jacquemin, D.; Moore, B.; Planchat, A.; Adamo, C.; Autschbach, J. Performance of an Optimally Tuned Range-Separated Hybrid Functional for 0 - 0 Electronic Excitation Energies. J. Chem. Theory Comput. 2014, 10, 1677-1685.

(78) Moore, B.; Charaf-Eddin, A.; Planchat, A.; Adamo, C.; Autschbach, J.; Jacquemin, D. Electronic Band Shapes Calculated with Optimally Tuned Range-Separated Hybrid Functionals. J. Chem. Theory Comput. 2014, 10, 4599-4608.

(79) Anderson, L. N.; Oviedo, M. B.; Wong, B. M. Accurate Electron Affinities and Orbital Energies of Anions from a Nonempirically Tuned Range-Separated Density Functional Theory Approach. J. Chem. Theory Comput. 2017, 13, 1656-1666.

(80) Gallandi, L.; Marom, N.; Rinke, P.; Körzdörfer, T. Accurate Ionization Potentials and Electron Affinities of Acceptor Molecules II: Non-Empirically Tuned Long-Range Corrected Hybrid Functionals. J. Chem. Theory Comput. 2016, 12, 605-614.

(81) Manna, D.; Blumberger, J.; Martin, J. M. L.; Kronik, L. Prediction of Electronic Couplings for Molecular Charge Transfer Using Optimally Tuned Range-Separated Hybrid Functionals. Mol. Phys. 2018, 116, 2497-2505.

(82) Liu, Q.; Wang, J.; Du, P.; Hu, L.; Zheng, X.; Chen, G. Improving the Performance of Long-Range-Corrected Exchange-Correlation Functional with an Embedded Neural Network. J. Phys. Chem. A 2017, 121, 7273-7281. 
(83) Wang, C.; Zhang, Q. Understanding Solid-State Solvation-Enhanced Thermally Activated Delayed Fluorescence Using a Descriptor-Tuned Screened Range-Separated Functional. J. Phys. Chem. C 2018, 123, 4407-4416.

(84) Fabrizio, A.; Meyer, B.; Corminboeuf, C. Machine Learning Models of the Energy Curvature Vs Particle Number for Optimal Tuning of Long-Range Corrected Functionals. J. Chem. Phys. 2020, 152, 154103.

(85) Vargas-Hernández, R. A. Bayesian Optimization for Calibrating and Selecting HybridDensity Functional Models. J. Phys. Chem. A 2020, 124, 4053-4061.

(86) Zheng, X.; Hu, L.; Wang, X.; Chen, G. A Generalized Exchange-Correlation Functional: The Neural-Networks Approach. Chem. Phys. Lett. 2004, 390, 186-192.

(87) Schneebeli, S. T.; Bochevarov, A. D.; Friesner, R. A. Parameterization of a B3LYP Specific Correction for Noncovalent Interactions and Basis Set Superposition Error on a Gigantic Data Set of $\operatorname{CCSD}(\mathrm{T})$ Quality Noncovalent Interaction Energies. J. Chem. Theory Comput. 2011, 7, 658-668.

(88) Snyder, J. C.; Rupp, M.; Hansen, K.; Müller, K.-R.; Burke, K. Finding Density Functionals with Machine Learning. Phys. Rev. Lett. 2012, 108, 253002.

(89) Li, L.; Baker, T. E.; White, S. R.; Burke, K. Pure Density Functional for Strong Correlation and the Thermodynamic Limit from Machine Learning. Phys. Rev. B 2016, 94, 245129.

(90) Li, L.; Snyder, J. C.; Pelaschier, I. M.; Huang, J.; Niranjan, U.-N.; Duncan, P.; Rupp, M.; Müller, K.-R.; Burke, K. Understanding Machine-Learned Density Functionals. Int. J. Quantum Chem. 2016, 116, 819-833.

(91) Seino, J.; Kageyama, R.; Fujinami, M.; Ikabata, Y.; Nakai, H. Semi-Local Machine- 
Learned Kinetic Energy Density Functional with Third-Order Gradients of Electron Density. J. Chem. Phys. 2018, 148, 241705.

(92) Grisafi, A.; Fabrizio, A.; Meyer, B.; Wilkins, D. M.; Corminboeuf, C.; Ceriotti, M. Transferable Machine-Learning Model of the Electron Density. ACS Cent. Sci. 2019, $5,57-64$.

(93) Schmidt, J.; Benavides-Riveros, C. L.; Marques, M. A. L. Machine Learning the Physical Nonlocal Exchange-Correlation Functional of Density-Functional Theory. J. Phys. Chem. Lett. 2019, 10, 6425-6431.

(94) Snyder, J. C.; Rupp, M.; Hansen, K.; Blooston, L.; Müller, K.-R.; Burke, K. OrbitalFree Bond Breaking via Machine Learning. J. Chem. Phys. 2013, 139, 224104.

(95) Bogojeski, M.; Vogt-Maranto, L.; Tuckerman, M. E.; Müller, K.-R.; Burke, K. Quantum Chemical Accuracy from Density Functional Approximations via Machine Learning. Nat. Comm. 2020, 11, 1-11.

(96) Wang, C.-I.; Joanito, I.; Lan, C.-F.; Hsu, C.-P. Artificial neural networks for predicting charge transfer coupling. J. Chem. Phys. 2020, 153, 214113.

(97) Ramakrishnan, R.; Hartmann, M.; Tapavicza, E.; von Lilienfeld, O. A. Electronic spectra from TDDFT and machine learning in chemical space. J. Chem. Phys. 2015, $143,084111$.

(98) Westermayr, J.; Marquetand, P. Machine Learning for Electronically Excited States of Molecules. In press in Chem. Rev.

(99) Häse, F.; Valleau, S.; Pyzer-Knapp, E.; Aspuru-Guzik, A. Machine Learning Exciton Dynamics. Chem. Sci. 2016, 7, 5139-5147.

(100) Westermayr, J.; Faber, F. A.; Christensen, A. S.; von Lilienfeld, O. A.; Marquetand, P. Neural Networks and Kernel Ridge Regression for Excited States Dynamics of 
$\mathrm{CH}_{2} \mathrm{NH}_{2}{ }^{+}$: From Single-State to Multi-State Representations and Multi-Property Machine Learning Models. Machine Learning: Science and Technology 2020, 1, 025009.

(101) Hachmann, J.; Olivares-Amaya, R.; Atahan-Evrenk, S.; Amador-Bedolla, C.; SánchezCarrera, R. S.; Gold-Parker, A.; Vogt, L.; Brockway, A. M.; Aspuru-Guzik, A. The Harvard Clean Energy Project: Large-Scale Computational Screening and Design of Organic Photovoltaics on the World Community Grid. J. Phys. Chem. Lett. 2011, 2, $2241-2251$.

(102) Hachmann, J.; Olivares-Amaya, R.; Jinich, A.; Appleton, A. L.; BloodForsythe, M. A.; Seress, L. R.; Román-Salgado, C.; Trepte, K.; Atahan-Evrenk, S.; Er, S. et al. Lead Candidates for High-Performance Organic Photovoltaics from HighThroughput Quantum Chemistry-the Harvard Clean Energy Project. Energy Environ. Sci. 2014, 7, 698-704.

(103) Democratizing Deep-Learning for Drug Discovery, Quantum Chemistry, Materials Science and Biology. https://github.com/deepchem/deepchem, 2016.

(104) Ju, C.-W.; Bai, H.; Li, B.; Liu, R. Machine Learning Enables Highly Accurate Predictions of PhotoPhysical Properties of Organic Fluorescent Materials: Emission Wavelengths and Quantum Yields. J. Chem. Inf. Model. 2021, 61, 1053-1065.

(105) Lopez, S. A.; Pyzer-Knapp, E. O.; Simm, G. N.; Lutzow, T.; Li, K.; Seress, L. R.; Hachmann, J.; Aspuru-Guzik, A. The Harvard Organic Photovoltaic Dataset. Sci. Data 2016, 3, 160086.

(106) Gómez-Bombarelli, R.; Aguilera-Iparraguirre, J.; Hirzel, T. D.; Duvenaud, D.; Maclaurin, D.; Blood-Forsythe, M. A.; Chae, H. S.; Einzinger, M.; Ha, D.-G.; Wu, T. et al. Design of Efficient Molecular Organic Light-Emitting Diodes by a High-Throughput Virtual Screening and Experimental Approach. Nat. Mater. 2016, 15, 1120-1127. 
(107) Yap, C. W. PaDEL-Descriptor: An Open Source Software to Calculate Molecular Descriptors and Fingerprints. J. Comput. Chem. 2011, 32, 1466-1474.

(108) Yang, Q.; Li, Y.; Yang, J.-D.; Liu, Y.; Zhang, L.; Luo, S.; Cheng, J.-P. Holistic Prediction of the $\mathrm{p} K_{a}$ in Diverse Solvents Based on a Machine-Learning Approach. Angew. Chem. Int. Ed. 2020, 59, 19282-19291.

(109) Landrum, G. RDKit: Open-Source Cheminformatics Software. 2016; https:// github.com/rdkit/rdkit/releases/tag/Release_2016_09_4.

(110) Rogers, D.; Hahn, M. Extended-Connectivity Fingerprints. J. Chem. Inf. Model. 2010, $50,742-754$.

(111) Willighagen, E. L.; Mayfield, J. W.; Alvarsson, J.; Berg, A.; Carlsson, L.; Jeliazkova, N.; Kuhn, S.; Pluskal, T.; Rojas-Chertó, M.; Spjuth, O. et al. The Chemistry Development Kit (CDK) v2.0: Atom Typing, Depiction, Molecular Formulas, and Substructure Searching. J. Cheminformatics 2017, 9, 1-19.

(112) Grimme, S.; Bannwarth, C.; Shushkov, P. A Robust and Accurate Tight-Binding Quantum Chemical Method for Structures, Vibrational Frequencies, and Noncovalent Interations of Large Molecular Systems Parametrized for All spd-Block Elements ( $Z=$ 1 - 86). J. Chem. Theory Comput. 2017, 13, 1989-2009.

(113) Bannwarth, C.; Ehlert, S.; Grimme, S. GFN2-xTB-An Accurate and Broadly Parametrized Self-Consistent Tight-Binding Quantum Chemical Method with Multipole Electrostatics and Density-Dependent Dispersion Contributions. J. Chem. Theory Comput. 2019, 15, 1652-1671.

(114) Pracht, P.; Caldeweyher, E.; Ehlert, S.; Grimme, S. A Robust Non-Self-Consistent Tight-Binding Quantum Chemistry Method for Large Molecules. 2019, 
(115) Chen, T.; Guestrin, C. XgBoost: A Scalable Tree Boosting System. Proceedings of the 22nd ACM SIGKDD International Conference on Knowledge Discovery and Data Mining. 2016; pp 785-794.

(116) Wolpert, D. H. Stacked Generalization. Neural Netw. 1992, 5, 241-259.

(117) Breiman, L. Stacked Regressions. Mach. Learn. 1996, 24, 49-64.

(118) Opitz, D.; Maclin, R. Popular Ensemble Methods: An Empirical Study. J. Artif. Intell. Res. 1999, 11, 169-198.

(119) Polikar, R. Ensemble Based Systems in Decision Making. IEEE Circuits Syst. Mag. 2006, 6, 21-45.

(120) Rokach, L. Ensemble-Based Classifiers. Artif. Intell. Rev. 2010, 33, 1-39.

(121) Coscrato, V.; de Almeida Inácio, M. H.; Izbicki, R. The NN-Stacking: Feature Weighted Linear Stacking through Neural Networks. Neurocomputing 2020, 399, 141152.

(122) Tibshirani, R. Regression Shrinkage and Selection via the Lasso. J. R. Stat. Soc. Series B Stat. Methodol. 1996, 58, 267-288.

(123) Kukreja, S. L.; Löfberg, J.; Brenner, M. J. A Least Absolute Shrinkage and Selection Operator (LASSO) for Nonlinear System Identification. IFAC Proc. Vol. 2006, 39, 814-819.

(124) Breiman, L.; Friedman, J.; Stone, C. J.; Olshen, R. A. Classification and Regression Trees; CRC Press, 1984.

(125) Svetnik, V.; Liaw, A.; Tong, C.; Culberson, J. C.; Sheridan, R. P.; Feuston, B. P. Random Forest: a Classification and Regression Tool for Compound Classification and QSAR Modeling. J. Chem. Inform. Comput. Sci. 2003, 43, 1947-1958. 
(126) Friedman, J. H. Stochastic Gradient Boosting. Comput. Stat. Data Anal. 2002, 38, $367-378$.

(127) Ke, G.; Meng, Q.; Finley, T.; Wang, T.; Chen, W.; Ma, W.; Ye, Q.; Liu, T.-Y. LightGBM: A Highly Efficient Gradient Boosting Decision Tree. Adv. Neural Inf. Process. Syst. 2017, 30, 3146-3154.

(128) Saunders, C.; Gammerman, A.; Vovk, V. Ridge Regression Learning Algorithm in Dual Variables. 15th International Conference on Machine Learning (ICML '98). 1998; pp $515-521$.

(129) Vovk, V. Statistical Learning Theory; John Wiley \& Sons, 1998.

(130) Cristianini, N.; Shawe-Taylor, J. An Introduction to Support Vector Machines and Other Kernel-based Learning Methods; Cambridge University Press, 2000.

(131) Vovk, V. Kernel Ridge Regression. In Empirical Inference: Festschrift in Honor of Vladimir N. Vapnik; Schölkopf, B., Luo, Z., Vovk, V., Eds.; Springer Berlin Heidelberg: Berlin, Heidelberg, 2013; pp 105-116.

(132) Vapnik, V. Pattern Recognition Using Generalized Portrait Method. Autom. Remote Control 1963, 24, 774-780.

(133) Boser, B. E.; Guyon, I. M.; Vapnik, V. N. A Training Algorithm for Optimal Margin Classifiers. Proceedings of the Fifth Annual Workshop on Computational Learning Theory. New York, NY, USA, 1992; p 144-152.

(134) Guyon, I.; Boser, B.; Vapnik, V. Automatic Capacity Tuning of Very Large VCDimension Classifiers. Advances in Neural Information Processing Systems. 1993; pp $147-155$.

(135) Cortes, C.; Vapnik, V. Support-Vector Networks. Mach. Learn. 1995, 20, 273-297. 
(136) Smola, A. J.; Schölkopf, B. A Tutorial on Support Vector Regression. Stat. Comput. 2004, 14, 199-222.

(137) Refaeilzadeh, P.; Tang, L.; Liu, H. Cross-Validation. In Encyclopedia of Database Systems; LIU, L., ÖZSU, M. T., Eds.; Springer US: Boston, MA, 2009; pp 532-538.

(138) Efron, B.; Hastie, T.; Johnstone, I.; Tibshirani, R. Least Angle Regression. Ann. Statist. 2004, 32, 407-499.

(139) Glantz, S.; Slinker, B. Primer of Applied Regression \& Analysis of Variance, ed; McGraw-Hill, Inc., New York, 2001.

(140) Ju, C.-W.; French, E. J.; Geva, N.; Kohn, A. W.; Lin, Z. Stacked Ensemble Machine Learning for Range-Separation Parameters. https://github.com/zhoulinumass/ ML-wPBE, 2021.

(141) Lin, Z.; Van Voorhis, T. Triplet Tuning: A Novel Family of Non-Empirical ExchangeCorrelation Functionals. J. Chem. Theory Comput. 2019, 15, 1226-1241.

(142) Zhao, Y.; Truhlar, D. G. The M06 Suite of Density Functionals for Main Group Thermochemistry, Thermochemical Kinetics, Noncovalent Interations, Excited States, and Transition Elements: Two New Functionals and Systematic Testing of Four M06Class Functionals and 12 Other Functionals. Theor. Chem. Acc. 2008, 120, 215-241.

(143) Szabo, A.; Ostlund, N. S. Modern Quantum Chemistry: Introduction to Advanced Electronic Structure Theory; Courier Corporation, 2012.

(144) Perdew, J. P.; Burke, K.; Ernzerhof, M. Generalized Gradient Approximation Made Simple. Phys. Rev. Lett. 1996, 77, 3865.

(145) Dirac, P. A. M. The Quantum Theory of the Emission and Absorption of Radiation. Proc. R. Soc. Lond. A 1927, 114, 243-265. 
(146) Ali, A.; Rafiq, M. I.; Zhang, Z.; Cao, J.; Geng, R.; Zhou, B.; Tang, W. TD-DFT Benchmark for UV-Visible Spectra of Fused-Ring Electron Acceptors Using Global and Range-Separated Hybrids. Phys. Chem. Chem. Phys. 2020, 22, 7864-7874.

(147) Alipour, M.; Safari, Z. Photophysics of OLED Materials with Emitters Exhibiting Thermally Activated Delayed Fluorescence and Used in Hole/Electron Transporting Layer from Optimally Tuned Range-Separated Density Functional Theory. J. Phys. Chem. C 2018, 123, 746-761.

(148) Gong, J.; Lam, J. W. Y.; Tang, B. Z. Benchmark and Parameter Tuning of Hybrid Functionals for Fast Calculation of Excitation Energies of AIEgens. Phys. Chem. Chem. Phys. 2020, 22, 18035-18039.

(149) Alipour, M.; Damiri, S. Unveiling the Role of Short-Range Exact-like Exchange in the Optimally Tuned Range-Separated Hybrids for Fuorescence Lifetime Modeling. J. Chem. Phys. 2020, 152, 204301.

(150) Runge, E.; Gross, E. K. U. Density-Functional Theory for Time-Dependent Systems. Phys. Rev. Lett. 1984, 52, 997-1000.

(151) Petersilka, M.; Gossmann, U. J.; Gross, E. K. U. Excitation Energies from TimeDependent Density-Functional Theory. Phys. Rev. Lett. 1996, 76, 1212-1215.

(152) van Leeuwen, R. Causality and Symmetry in Time-Dependent Density-Functional Theory. Phys. Rev. Lett. 1998, 80, 1280-1283.

(153) de Silva, P.; Kim, C. A.; Zhu, T.; Van Voorhis, T. Extracting Design Principles for Efficient Thermally Activated Delayed Fluorescence (TADF) from a Simple Four-State Model. Chem. Mater. 2019, 31, 6995-7006.

(154) Riehl, J. P.; Richardson, F. S. Circularly Polarized Luminescence Spectroscopy. Chem. Rev. 1986, 86, 1-16. 
(155) Qiu, Z.; Ju, C.-W.; Frédéric, L.; Hu, Y.; Schollmeyer, D.; Pieters, G.; Müllen, K.; Narita, A. Amplification of Dissymmetry Factors in $\pi$-Extended [7]- and [9]Helicenes. J. Am. Chem. Soc. 2021, 143, 4661-4667.

(156) Li Manni, G.; Carlson, R. K.; Luo, S.; Ma, D.; Olsen, J.; Truhlar, D. G.; Gagliardi, L. Multiconfiguration Pair-Density Functional Theory. J. Chem. Theory Comput. 2014, 10, 3669-3680.

(157) Kaduk, B.; Kowalczyk, T.; Van Voorhis, T. Constrained Density Functional Theory. Chem. Rev. 2012, 112, 321-370.

(158) Zhu, Y.; Herbert, J. M. Self-Consistent Predictor/Corrector Algorithms for Stable and Efficient Integration of the Time-Dependent Kohn-Sham Equation. J. Chem. Phys. 2018, 148, 044117.

(159) Zhu, Y.; Alam, B.; Herbert, J. Broadband X-Ray Absorption Spectra from TimeDependent Kohn-Sham Calculations. ChemRxiv 2021, 


\section{TOC Graphic}

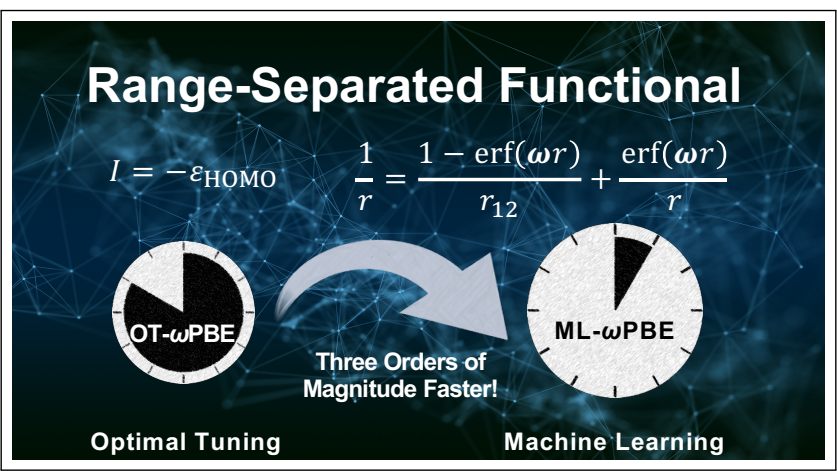

